STEM, STEAM, STREAM:

Posibilidades, reflexiones y experiencias

\title{
Una experiencia formativa con BlocksCAD con futuros docentes de matemáticas en secundaria
}

Recepción: 20/11/2020 | Revisión: 17/05/2021 | Aceptación: 05/08/2021 | Publicación: 01/10/2021

Pablo BELTRÁN-PELLICER

Universidad de Zaragoza

pbeltran@unizar.es

https://orcid.org/0000-0002-1275-9976
José M. MUÑOZ-ESCOLANO

Universidad de Zaragoza

jmescola@unizar.es

https://orcid.org/oooo-0002-8713-4591

Resumen: Ciertas herramientas de software empleadas en los procesos de modelado y fabricación de piezas en 3D ofrecen una excelente oportunidad para el desarrollo de conocimientos y competencias matemáticas, a la vez que permiten establecer conexiones inter e intra-disciplinares. En este artículo se describe una experiencia con 22 futuros profesores de secundaria, estudiantes del máster de profesorado de la especialidad de Matemáticas. La tarea propuesta a los participantes fue el modelado de un dado, considerando aquellos factores que pueden afectar a su sesgo. Para ello, se usó BlocksCAD, entorno de programación por bloques que permite describir las piezas mediante primitivas geométricas, transformaciones y operaciones lógicas. Una vez terminado el modelado, se pidió a los participantes que plantearan secuencias didácticas que establecieran las conexiones mencionadas. Se recogieron y analizaron las producciones escritas de los participantes, así como los archivos xml con la construcción de los dados. Entre los resultados, se han observado tres tipos diferentes de modelado, así como una gran variedad de uso de los bloques. El establecimiento de conexiones en las secuencias didácticas es, en general, escaso, lo cual abre la puerta a la necesidad de experiencias similares donde se expliciten conexiones con otras materias.

Palabras clave: educación matemática; STEM; pensamiento computacional; impresión 3D.

\section{A TRAINING EXPERIENCE USING BLOCKSCAD WITH FUTURE HIGH SCHOOL TEACHERS OF MATHEMATICS}

Abstract: Some of the software tools used in the modelling and manufacturing processes of ${ }_{3} D$ objects and parts offer an excellent opportunity for the development of mathematical knowledge and skills, while allowing the establishment of inter and intra-disciplinary connections. This article describes an experience with 22 future secondary school teachers, students of the Master's Degree of Teaching in Secondary Education. The task proposed to the participants was the modelling of a dice considering those factors that may affect its bias, using the BlocksCAD block programming environment. This software allows for the description of parts using geometric primitives, transformations and logical operations. Once the modelling was finished, the participants were asked to propose didactical sequences that establish the aforementioned connections. The written productions of the participants were collected and analysed, as well as the xml files with the construction of the dice. The results show 
three different types of modelling, as well as a great variety when using the available blocks. The establishment of connections in the didactic sequences is, in general, weak, which opens the door to the need for similar experiences where connections with other subjects are made explicit.

Keywords: mathematics education; STEM; computational thinking; ${ }_{3} D$ printing.

\section{UNA EXPERIÈNCIA FORMATIVA AMB BLOCKSCAD AMB FUTURS DOCENTS DE MATEMÀTIQUES A SECUNDÀRIA}

Resum: Algunes eines de software utilitzades en els processos de modelatge i fabricació de peces en $3 D$ ofereixen una oportunitat excel-lent per al desenvolupament de coneixements $i$ competències matemàtiques, al mateix temps que permeten establir connexions inter i intra-disciplinars. En aquest article es descriu una experiència amb 22 futurs mestres de secundària, estudiants del màster de professorat de l'especialitat de Matemàtiques. Se'ls va proposar la tasca de modelatge d'un dau, considerant els factors que poden afectar el seu biaix. Es va utilitzar BlocksCAD, un entorn de programació per blocs que permet descriure les peces mitjançant primitives geomètriques, transformacions $i$ operacions lògiques. Un cop acabat el modelatge, es va demanar als participants que plantegessin seqüències didàctiques que establissin les connexions esmentades. Es van recollir i analitzar les produccions escrites dels participants, així com els arxius xml amb la construcció dels daus. Entre els resultats, s'observen tres tipus diferents de modelatge, així com una gran varietat de l'ús dels blocs. L'establiment de connexions en les seqüències didàctiques és, en general, escàs. Per tant, s'obre la porta a la necessitat d'experiències similars on s'explicitin connexions amb altres matèries.

Paraules clau: educació matemàtica; STEM; pensament computacional; impressió $3 D$.

\section{Introducción}

La utilización de las tecnologías de la información y la comunicación (TIC) para la enseñanza y aprendizaje ha acompañado a la educación matemática desde el auge de los ordenadores personales en los años 80 del siglo pasado (Hoyles y Lagrange, 2010). De aquella etapa, un claro representante es el lenguaje LOGO y su célebre tortuga (Papert, 1980), con el que se trabajaba el pensamiento computacional (aunque no se llamara así todavía) al mismo tiempo que se profundizaba en el contenido matemático con situaciones donde emergían contenidos de los diferentes bloques curriculares. La construcción de un reloj, por ejemplo, era algo que requería poner en juego diversas ideas del ámbito de la geometría. Actualmente, hablar de geometría, matemáticas y TIC es hablar de entorno de geometría dinámica, entre los que destaca la opción libre de GeoGebra. Hasta hace poco, estos programas solo posibilitaban el trabajo en geometría plana, por lo que la mayoría de las publicaciones existentes se orientan en esa dirección. Sin embargo, Gutiérrez y Jaime (2015) ya mencionan el potencial que ofrece la aparición de programas de geometría dinámica tridimensional, los cuales pueden suplir la carencia, muchas veces crónica, de materiales manipulativos apropiados para el aprendizaje de nociones de geometría espacial en los centros educativos.

Si bien GeoGebra, al igual que Cabri en su momento y otras, posee una orientación específica hacia las matemáticas, actualmente hay ciertas herramientas TIC pensadas para un ámbito puramente tecnológico o de diseño que plantean interesantes oportunidades para la educación matemática y para establecer conexiones inter e intra-disciplinares (Hohenwarter y Fuchs, 2004; Ortega, 2005). Entre estas herramientas se encuentran las que se utilizan dentro del flujo de modelado 
e impresión de piezas en 3D. Un ejemplo es el entorno de programación por bloques BlocksCAD, en el que se describen las piezas a modelar a partir de primitivas geométricas, operadores lógicos y transformaciones.

Dado el potencial didáctico de estas herramientas, resulta pertinente el diseño e implementación de experiencias formativas con futuros profesores de matemáticas. El objetivo de este artículo es describir una de tales experiencias, que conjuga diversas facetas de los conocimientos y competencias deseables en un docente de matemáticas y que permite establecer conexiones con otras disciplinas científico-tecnológicas. Además, analizamos las producciones finales de los participantes e indagamos en las dificultades percibidas por ellos y las propuestas de diseño de actividades de secundaria que fomenten conexiones con el tipo de actividades realizadas en la experiencia.

En primer lugar, describiremos los antecedentes y el marco teórico, donde se incluye una breve síntesis de la impresión 3D en educación, una sección dedicada al modelado de cuerpos en el entorno de programación por bloques de BlocksCAD y otra a los tipos de conexiones inter e intradisciplinares y con el entorno que posibilitan los proyectos y tareas STEM. Posteriormente, se describe la metodología empleada, así como el contexto en que desarrolló la experiencia, lo cual da paso a la presentación y discusión de los resultados obtenidos. Finalmente, se exponen las conclusiones más relevantes.

\section{Antecedentes y marco teórico \\ 1.1 Modelado e impresión en 3D}

Entre las herramientas TIC no específicas de matemáticas encontramos las dedicadas al modelado y la impresión en 3D. Las impresoras en 3D han visto disminuido su coste de tal manera (Jones y otros, 2011) que muchos centros educativos ya disponen de alguna para realizar actividades en asignaturas como Tecnología (González-Gómez, et al., 2012) y Matemáticas (Beltrán-Pellicer, 2017; Beltrán-Pellicer y Rodríguez-Jaso, 2017, 2018).

Las impresoras 3D aplicadas a la educación matemática constituyen un recurso que permite plantear actividades que implican aprendizaje por indagación o aprendizaje basado en la resolución de problemas (Wang et al., 2019) donde los estudiantes, en el rol de creadores, son capaces de, por ellos mismos, generar ideas innovadoras y producir objetos concretos empleando las TIC ( Ng, 2017; $\mathrm{Ng}$ y Chan, 2019), además de producir cambios positivos sobre sus concepciones acerca del error en los procesos de enseñanza y aprendizaje (Dickson et al., 2020).

Aunque hay varias técnicas de impresión 3D, las máquinas que podemos encontrar en los centros educativos fabrican los objetos capa a capa, de abajo hacia arriba. Existen repositorios en Internet, como GitHub o Thingiverse, donde es posible descargarse piezas ya listas para imprimir, incluso parametrizables, en formato STL, el cual describe la superficie o mallado del objeto. Y, más interesante para nuestros propósitos educativos, hay diferentes paquetes de software de diseño asistido por ordenador (CAD), que nos permiten crear nuestras propias piezas o modificar otras. Una primera clasificación de los CAD nos llevaría a distinguir, por un lado, entornos interactivos en donde se trabaja desde alguna perspectiva tridimensional, con mayor o menor grado de complejidad, en función de si se puede realizar acotaciones, considerar restricciones de diseño o incluir parámetros. 
Aquí encontraríamos, por ejemplo, 3D Builder de Microsoft, Rhinoceros 3D, Inventor, TinkerCAD (aplicación web gratuita) o la opción libre FreeCAD. Por otro lado, existen entornos CAD en los que se describe la pieza. Es el caso de OpenSCAD, el cual no es un editor interactivo sino un compilador 3D basado en un lenguaje de descripción textual que a su vez se basa en el uso de unas pocas primitivas geométricas, transformaciones y operaciones lógicas, a lo que se añaden instrucciones propias de un lenguaje de programación, como variables, iteraciones, módulos, etc. El fichero de texto resultante, una vez compilado, genera el modelo tridimensional. Es interesante destacar que las variables, que permiten parametrizar las piezas, son algo inherente a este tipo de modelado (Chytas et al., 2018). Por ejemplo, para modelar una tuerca, en OpenSCAD escribiríamos el código que describiría una tuerca de métrica m. Autores como Schelly et al. (2015) señalan que el manejo OpenSCAD es sencillo y que media hora de instrucción basta para empezar a modelar piezas sencillas. No obstante, hay algunos aspectos del entorno que requieren de una familiarización, como el empleo de la recursividad (Chytas et al., 2018).

Como OpenSCAD es software libre, han surgido otros entornos de modelado basados en él. Es interesante el caso de BlocksCAD, que ofrece una interfaz visual de programación por bloques similar a la de Scratch, solo que en este caso no estaríamos programando, sino describiendo el cuerpo a modelar. El sistema de bloques presenta una ventaja, sobre todo desde el punto de vista educativo (Beltrán-Pellicer et al., 2020), ya que elimina el obstáculo que podría suponer la sintaxis textual de OpenSCAD. Es decir, al describir la pieza no tenemos que preocuparnos de si hemos cometido un error de escritura (una llave mal puesta, una instrucción con erratas) porque el sistema de bloques no te permite cometerlos. Desde luego, sigue siendo posible que la pieza resultante no coincida con nuestra imagen mental de la misma, pero eso es otra cuestión. A pesar de que los programadores experimentados pueden percibir estos entornos como menos potentes y capaces, su transparencia y facilidad de uso permite que el alumnado los emplee directamente (Weintrop y Wilensky, 2015).

La similitud que presentan BlocksCAD y Scratch permiten hacer extensibles al primero algunas de las conclusiones que arrojan las recientes investigaciones sobre el potencial de la programación por bloques de Scratch como recurso didáctico para matemáticas. En este sentido, diversos estudios apuntan a que facilita la adquisición de contenidos matemáticos, al mismo tiempo que su orientación al pensamiento computacional permite desarrollar procesos propios del Pensamiento Matemático Avanzado (abstracción, generalización y formulación y validación de hipótesis) (BaezaAlba, Claros-Mellado y Sánchez-Campaña, 2016; Ferrer, 2011). Así mismo, es destacable que el empleo de Scratch incide positivamente en el dominio afectivo del alumnado hacia las matemáticas (Jorge-Pozo, Jiménez-Gestal y Murillo, 2017).

Al igual que Scratch, la conexión de BlocksCAD con el pensamiento computacional (Wing, 2006) es evidente. Si bien es algo que no aparece de forma explícita en el currículo actual, diversos autores (Baeza-Alba, et al., 2016), lo relacionan con la resolución de problemas y el planteamiento de investigaciones matemáticas, especialmente aquellas que involucran procesos de matematización y modelización. La inclusión de la programación por bloques en la formación inicial de profesores es un medio que permite conectar la enseñanza de las matemáticas desde la comprensión de los conceptos con el pensamiento computacional (Gleasman y Kim, 2020). 
En el caso de BlocksCAD, el modelado de piezas exige competencia en visualización espacial y el conocimiento de propiedades de cuerpos y transformaciones en el espacio. Además, dependiendo de la pieza en cuestión, se enlaza también con la geometría analítica, pues se pueden definir trayectorias mediante ecuaciones paramétricas. Se trata, en definitiva, de un recurso con un gran potencial para desarrollar diversos contenidos curriculares

\subsection{Conexiones inter e intra-disciplinares y con el entorno: STEM}

El informe Rocard (Rocard et al, 2007) señala la necesidad social de fomentar vocaciones científicotecnológicas entre el alumnado, empleando el acrónimo STEM. Posteriormente, surge el constructo "educación STEM" como la enseñanza integrada de las Ciencias, Tecnología, Ingeniería y Matemáticas para fomentar la alfabetización STEM en la ciudadanía. No obstante, Martín-Páez, Aguilera, Perales-Palacios y Vílchez-González (2019) señalan que la definición de educación STEM es controvertida y ha ido evolucionando a lo largo de estos años. Así, Shaughnessy (2013, p. 324) ofrece una definición de educación STEM, poniendo el énfasis en la M de su acrónimo: "la resolución de problemas que se basan en conceptos y procedimientos de las matemáticas y la ciencia, al tiempo que se incorpora el trabajo en equipo y la metodología de diseño de la ingeniería y se utiliza la tecnología adecuada".

Por otro lado, Martín-Páez et al. (2019) señalan que algunos autores inciden en la presencia en actividades STEM de, al menos, dos de estas disciplinas en las experiencias de clase mientras que otros inciden en que es necesario incluir las cuatro, y otros exigen un adecuado grado de integración de estas las disciplinas durante los procesos de enseñanza-aprendizaje.

Alsina (2020) relaciona la educación STEM con la educación matemática dentro del contexto más amplio de las conexiones para el aprendizaje de las matemáticas de larga tradición en el área (Freudhental, 1991; NCTM, 2002). Estas conexiones matemáticas pueden tener una triple naturaleza (Alsina, 2020):

- Conexiones intra-disciplinares: referidas a las relaciones entre los diferentes temas de contenido matemático y entre los contenidos y los procesos matemáticos.

- Conexiones interdisciplinares: las relaciones de las matemáticas con otras áreas de conocimiento.

- Conexiones con el entorno: las relaciones de las matemáticas con el entorno que nos rodea (enfoque globalizado).

En este caso, la educación STEM promovería el establecimiento de conexiones interdisciplinares significativas entre las Matemáticas y otras áreas como la Ciencia, la Tecnología o la Ingeniería.

En cuanto a su implementación en el aula, Stohlmann (2020) plantea una clasificación de tres grandes métodos para que los profesores de matemáticas en Secundaria puedan plantear la integración de las materias STEM: 
- Problemas de respuesta abierta a través de desafíos de diseño de ingeniería.

- Problemas de respuesta abierta a través de modelización matemática en contextos científicos.

- Problemas de respuesta abierta a través de matemáticas integradas con tecnología y de aprendizaje basado en juegos.

Respecto a esta clasificación de métodos, Bush, Cox y Cook (2016) plantean actividades o proyectos STEM que corresponden a la primera clase, donde los estudiantes de Secundaria diseñan objetos reales, en este caso, una mano ortopédica, que pueden ser modelizados por cuerpos geométricos empleando un software específico de diseño de objetos (TinkerCAD) y mediante el uso de impresoras 3D. Otras experiencias STEM similares a la anterior sobre la fabricación de objetos con impresoras 3D poniendo el foco en el ámbito de las matemáticas también han sido propuestas y estudiadas por Ng (2017) y Ng y Chang (2019). Diego-Mantecón, Arcera, Fernández-Blanco, y Lavicza (2019) estudian el cambio en las creencias de estudiantes que desarrollan un proyecto donde se construye un robot para resolver un cubo de Rubik. Este enfoque conjunto de resolución de problemas matemáticos junto con la ingeniería y la tecnología promueve creencias positivas sobre el aprendizaje de las matemáticas. Además de las creencias, un trabajo prolongado en proyectos STEM por parte de los estudiantes también ha acreditado una mejora en la adquisición de las competencias clave del currículo (Diego-Mantecón, Fernández-Blanco, Ortiz-Laso, y Lavicza, 2021).

McDonald (2016), en su revisión de investigaciones educativas, concluye que es necesaria una formación de profesorado de alta calidad ya que ésta influye positivamente en las actitudes y la motivación de los estudiantes hacia el STEM. Diego-Mantecón et al. ( 2021) también señalan que la escasa formación en educación STEM y enfoques interdisciplinares es una de las razones por las que los profesores rehúsan participar en proyectos STEM. Por otro, lado, también existen trabajos donde se presentan distintas experiencias sobre formación de profesorado de matemáticas en STEM (Carmona-Mesa, Cardona Zapata y Castrillón-Yepes, 2020).

Existen diferentes marcos teóricos que dividen los conocimientos y competencias del profesor de matemáticas en diferentes facetas: conocimientos matemáticos, conocimientos didácticomatemáticos, conocimientos y competencias tecnológicas, etc. (Godino, Batanero, Font y Giacomone, 2016; Mishra y Koehler, 2006). Es deseable que las propuestas que se desarrollan en los programas de formación inicial incluyan actividades que articulen todas ellas.

El objetivo que nos planteamos es explorar el conocimiento del contenido matemático que se pone en juego en una tarea de modelado 3D, en cuanto a geometría y visualización, así como las conexiones inter e intra-disciplinares, y con el entorno, que identifican los futuros docentes de secundaria.

\section{Metodología, contexto y descripción de la experiencia}

El estudio realizado es de carácter exploratorio, adoptando una metodología cualitativa de tipo descriptivo e interpretativo (Hernández, Fernández y Baptista, 2010) basada en el análisis de las producciones de los participantes, tanto los informes escritos como los ficheros xml con el modelado realizado en BlocksCAD. A continuación, presentamos brevemente el entorno de programación de 
BlocksCAD y describimos el contexto y la experiencia llevada a cabo con los participantes.

\subsection{Modelado de cuerpos en el entorno de programación por bloques de BlocksCAD}

En el análisis de resultados haremos referencia a las primitivas geométricas y demás funcionalidades que ofrece BlocksCAD, por lo que resulta pertinente dedicar una sección del marco metodológico a describir brevemente el entorno. Como ya hemos mencionado, los bloques de BlocksCAD presentan una correspondencia directa con las instrucciones de texto propias de OpenSCAD. De hecho, el propio software nos permite ver el código que se va generando.

Los bloques disponibles se agrupan en un menú por categorías: primitivas (3D y 2D), operaciones con conjuntos, transformaciones geométricas, operaciones matemáticas (cálculos), lógicas, algorítmicas y herramientas de texto. A lo largo del artículo, para facilitar la distinción entre los objetos de BlocksCAD y los correspondientes cuerpos y transformaciones en el espacio, nos referiremos a ellos con sus nombres originales en inglés, aunque desde la versión 1.8.3 ya hay una versión traducida al español.

Las primitivas 3D son cube, cylinder, sphere y torus. Aunque el término cubo designa en geometría un caso concreto de prisma (prisma cuadrado con altura igual al lado de la base), en BlocksCAD la primitiva cube modela un prisma rectangular de dimensiones arbitrarias. Esto se aprecia en la Figura 1, donde además observamos que se puede elegir entre modelarlo centrado (con centro en el origen de coordenadas) o no centrado (con un vértice en el origen, de manera que el modelo se extiende en la dirección positiva de cada eje).

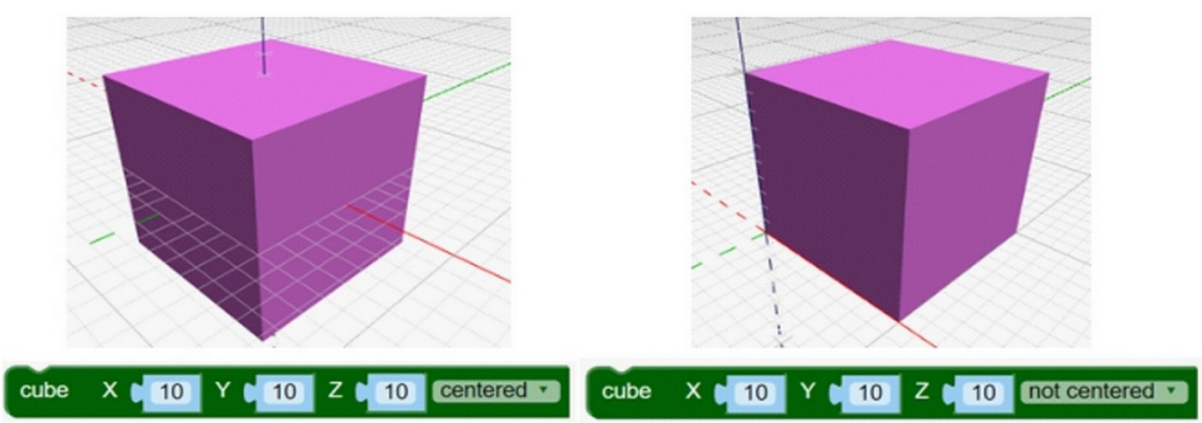

Figura 1. cube(10,10,10) para modelar un cubo de $10 \mathrm{~mm}$ de arista.

La primitiva cylinder ofrece más parámetros de los necesarios para especificar un cilindro. Además del radio de la base y la altura, aparece un segundo radio, un candado y la posibilidad de centrar o no el modelo. Si el candado está cerrado, ambos radios serán iguales, mientras que si el candado está abierto podremos especificar el radio de la base inferior y el radio de la base superior por separado. Esto nos permite modelar directamente un tronco de cono y, si uno de los radios es nulo, un cono. Las otras primitivas $3 \mathrm{D}$ son sphere y torus, que permiten modelar una esfera y un toro, respectivamente. 
En el apartado del menú dedicado a las operaciones con conjuntos tenemos la unión (union), intersección (intersection), diferencia (difference) y el operador hull, con el que se obtiene la envoltura convexa. Estos bloques actúan como operadores sobre objetos creados ya con anterioridad, y se asume que las primitivas 3D modelan cuerpos macizos. De esta manera, union crea un objeto formado por todos los puntos que están en alguno de los objetos sobre los que se aplica; intersection, con los puntos que están en todos y cada uno de los objetos; y difference crea un nuevo objeto compuesto por todos los puntos que están en el primer objeto y no están en los demás. Conviene observar que no son operadores binarios.

Dentro de la categoría de transformaciones, encontramos los bloques necesarios para hacer traslaciones, rotaciones, simetrías, escalados, escalados a lo largo de un eje, cambios de color, extrusiones y la elección del número de lados. Las extrusiones permiten modelar objetos con sección transversal definida y fija a lo largo de una línea (extrusión lineal) o en torno a un eje (extrusión rotacional). Se emplean, por ejemplo, cuando se quiere modelar una pieza extruyendo su planta. El bloque sides es esencial para poder aumentar el rango de cuerpos que se pueden modelar con las primitivas. Así, si lo que queremos es modelar un prisma, lo haremos aplicando el bloque sides a un cylinder. De esta manera, el cilindro ideal pasará a ser modelado por un prisma poligonal inscrito con el número de lados que especifique sides (ver el prisma hexagonal de la Figura 2).
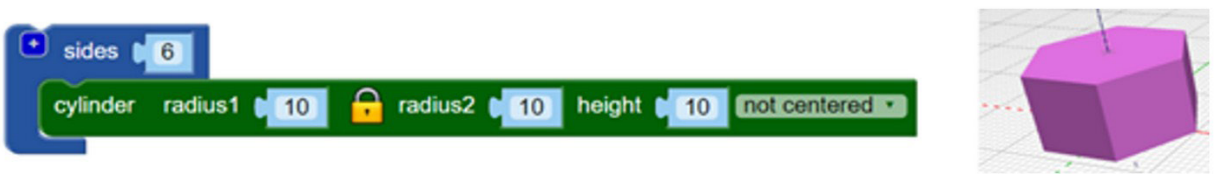

Figura 2. Modelado de un prisma recto hexagonal aplicando la transformación sides(6) sobre un cylinder.

\subsection{Descripción de la experiencia}

La experiencia se llevó a cabo con 22 futuros profesores de matemáticas, estudiantes del Máster de Profesorado de Secundaria y Bachillerato, en una sesión de clase de tres horas de duración. Ambos autores participaron como profesores-investigadores. Ninguno de los participantes había tenido contacto con BlocksCAD anteriormente, aunque uno de ellos manifestó que había utilizado OpenSCAD. En primer lugar, se dedicó aproximadamente una hora a la realización de un taller ${ }^{2}$, con el objetivo de que los participantes se familiarizasen mínimamente con el entorno y las aplicaciones a la impresión en 3D. Dicho taller incluyó una revisión de las primitivas básicas de BlocksCAD y el modelado de piezas (Figura 3).
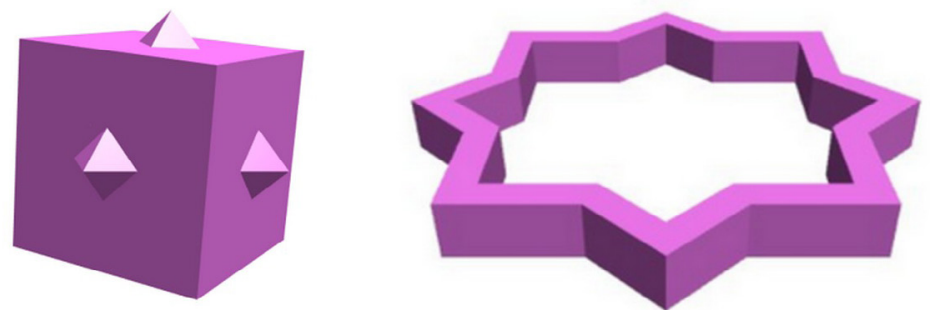

Figura 3. Ejemplos de piezas modeladas en el taller introductorio. Fuente: Beltrán-Pellicer, Rodríguez-Jaso y Muñoz-Escolano (2020).

2 El taller puede visitarse en: https://mat3d.github.io/. 
Una vez recogida la ficha correspondiente a la Actividad 1, se agrupó a los participantes por parejas o grupos de tres para que trabajasen en el diseño del dado, lo que constituyó la Actividad 2. Los diseños fueron recogidos en formato $\mathrm{xml}$, con el fin de poder analizar más adelante cómo fueron las construcciones. Además, los participantes tuvieron que rellenar, individualmente, una ficha a partir de la siguiente consigna:

Haz una descripción verbal por escrito del procedimiento seguido en BlocksCAD para modelar el dado. No puedes apoyarte en un esbozo de los bloques. Describe los problemas que habéis tenido.

Estas dos primeras actividades tienen como objetivo poner en juego el conocimiento matemático. Se planteó una Actividad 3, en grupos pequeños, orientada a indagar en la competencia de diseño de actividades y establecimiento de conexiones inter e intra-disciplinares:

Diseña una secuencia de actividades para secundaria inspirada en esta tarea, estableciendo conexiones intra y extra-matemáticas.

De esta manera, la experiencia consta de la estructura que se indica en la Tabla 1.

\begin{tabular}{lccc} 
& $\begin{array}{c}\text { Duración } \\
\text { aproximad } \\
\text { a } \\
\text { (minutos) }\end{array}$ & Observaciones & Tipo de producción recogida \\
\hline $\begin{array}{l}\text { Taller } \\
\text { introductorio }\end{array}$ & 60 & En grupos de 2 o 3, con ordenador. & Ninguna. \\
Actividad 1 & 20 & Individualmente y sin ordenador & Informe escrito. \\
Actividad 2 & 80 & En grupos de 2 o 3, con ordenador. & Informe escrito y fichero xml. \\
Actividad 3 & 20 & En grupos de 2 o 3, sin ordenador. & Informe escrito. \\
\hline
\end{tabular}

Tabla 1. Estructura de la experiencia.

\section{Resultados y discusión}

A continuación, presentamos los resultados obtenidos en lo que se refiere al diseño de los dados y los tipos de actividades propuestas inspiradas en esta tarea. Nos referiremos a las producciones de cada uno de los nueve equipos como Ei.

\subsection{Modelado de los dados por los futuros profesores}

Como se ha señalado en la descripción de la tarea, entre la realización de la tarea consistente en esbozar el proceso de modelado del dado y el modelado con la herramienta BlocksCAD, se señaló que un aspecto interesante a considerar era la redondez del dado. Encontramos tres grandes tipos de solución para el modelado del cuerpo del dado: 


\section{Modelado de tipo A}

Siete de los nueve equipos optaron por modelar el dado como un cubo que se interseca con una esfera (E2, E3, E4, E6, E7, E8, E9).
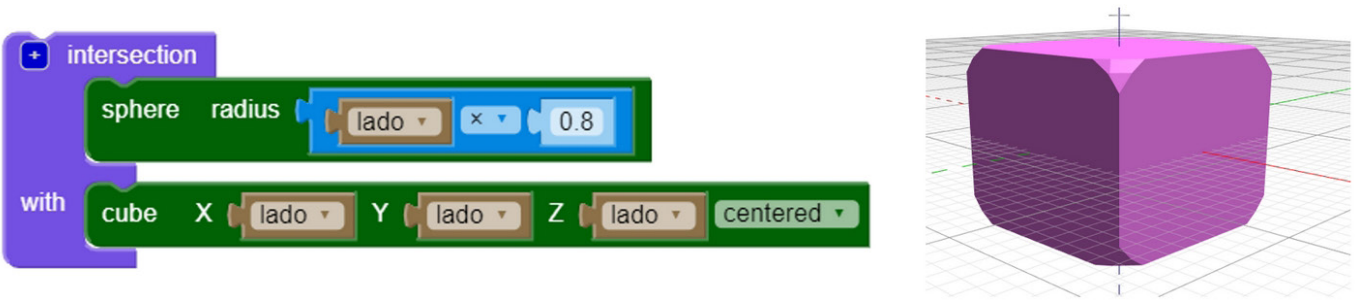

Figura 4. Modelado del cuerpo del dado como la intersección de un cubo y una esfera.

\section{Modelado de tipo B}

Uno de los equipos (E1) modeló el dado como la diferencia de un cubo con una corona esférica, resultante esta última de hacer, a su vez, la diferencia entre dos esferas concéntricas.
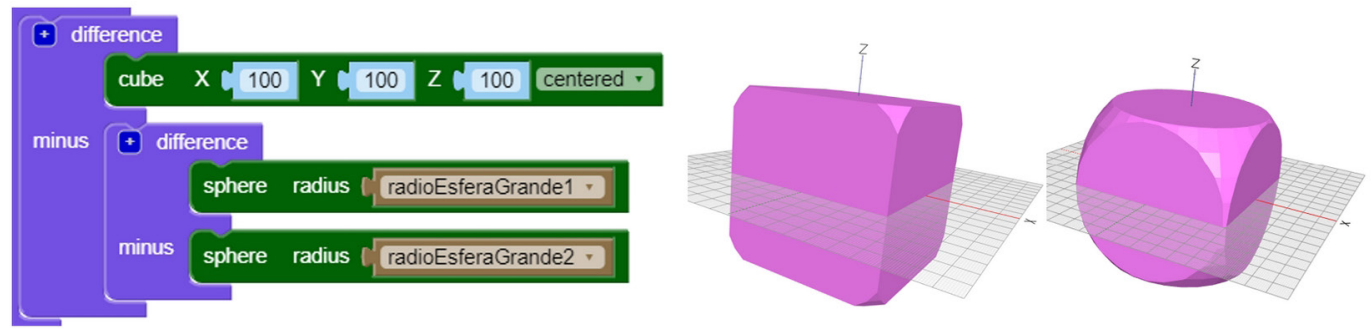

Figura 5. Modelado del cuerpo del dado como la diferencia de un cubo y una corona esférica.

\section{Modelado de tipo C}

Finalmente, uno de los equipos (E5), planteó el modelado del dado como la intersección de tres cilindros y una esfera. El primero de los cilindros tiene su eje en el eje $Z$, mientras que el segundo es ese mismo cilindro rotado $90^{\circ}$ en el eje $X$ y para el tercero se procede rotando el primer cilindro $90^{\circ}$ en el eje $Y$. De esta manera, consiguen tres cilindros, con sus ejes ubicados en los ejes de coordenadas, pudiendo ajustar el biselado de las aristas del "cubo" que conforma su intersección. Posteriormente, intersecan con una esfera para poder suavizar las esquinas. En la Figura 6 se observan diferentes renderizaciones de este modelo. 


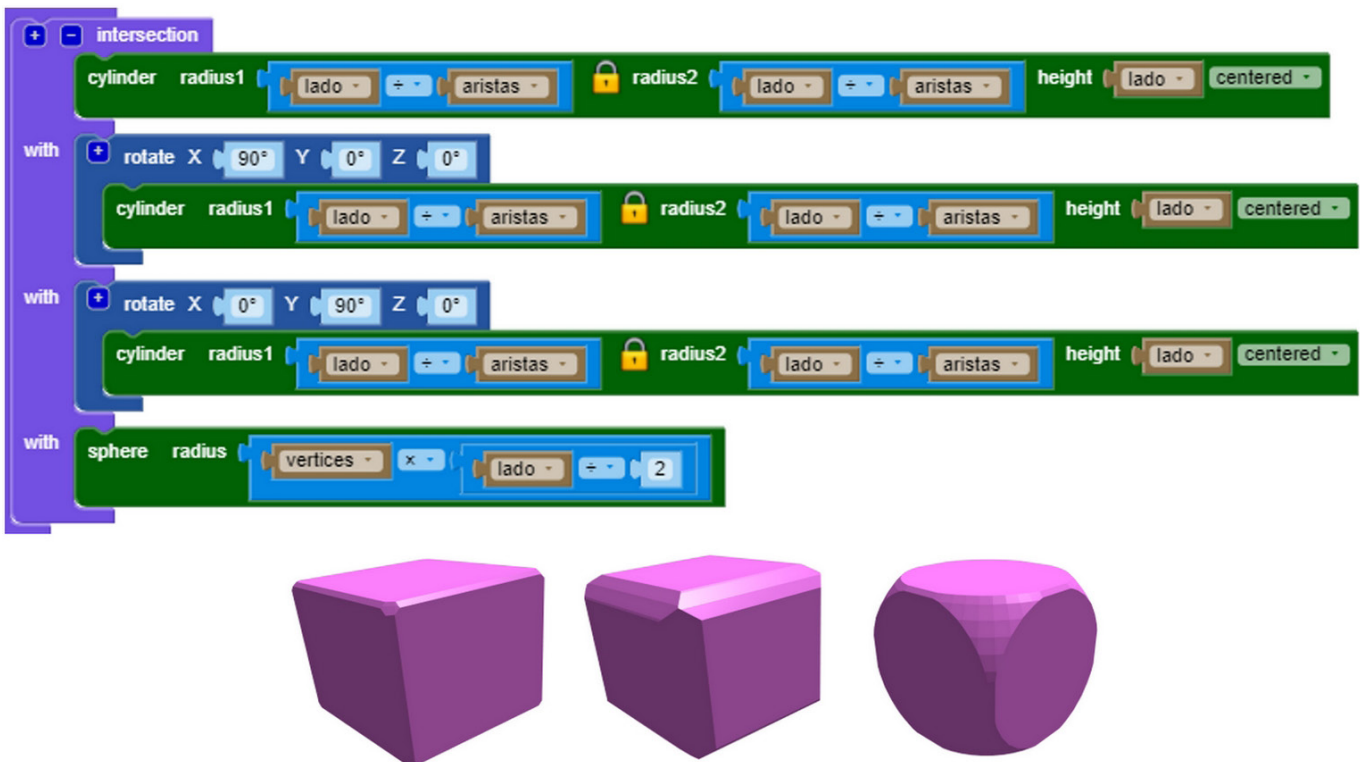

Figura 6. Modelado del cuerpo del dado como la intersección de tres cilindros y una esfera.

En cuanto a la forma de representar el valor de cada cara, también vemos diferentes opciones. Todos los equipos, menos el E2, han modelado estos valores utilizando la diferencia entre el cuerpo del dado y esferas, convenientemente situadas, con el objetivo de modelar cavidades. El equipo E2, en cambio, modeló el valor a partir de cilindros salientes (Figura 7).

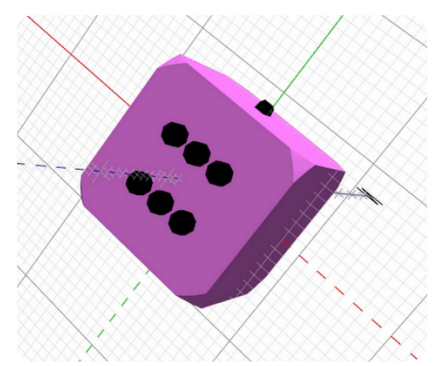

Figura 7. Representación de los valores de cada cara por medio de cilindros salientes (E2). Caras opuestas no equilibradas.

La estrategia más básica para tratar de obtener un buen dado; es decir, que no esté sesgado, consiste en equilibrar las caras opuestas. Es por esta razón que las caras opuestas de los dados estándar suman siete. De esta manera, se retira la misma cantidad de material en las tres parejas de caras que componen el cuerpo del dado. Casi todos los equipos siguieron esta estrategia, con la salvedad del E2, cuyo diseño está incompleto y no es posible saberlo; y el E4, cuyas parejas de caras opuestas son $2-1,5-6$ y 3-4.

Otro aspecto clave a considerar es el volumen retirado en cada cara. Aquí también se distinguen diversas estrategias: 
Parametrizar el radio una esfera (la de la cara del uno), y realizar cálculos para el radio de las demás, con el objetivo de igualar el volumen retirado de cada cara.

Parametrizar, por caras, el radio de las esferas de cada cara. Tras una experimentación con el dado real, o unos cálculos más exhaustivos, podría terminar de ajustarse.

Sin parametrizar, pero considerando diferentes radios.

En cuanto a las herramientas utilizadas para distribuir las cavidades en cada cara, se distinguen tres tipos de soluciones. Hay tres equipos que únicamente emplean traslaciones (E1, E4, E8); otros tres (E2, E3, E9) que emplean traslaciones y rotaciones; y finalmente, hay tres equipos (E5, E6, E7) que emplean traslaciones, rotaciones y simetrías (mirror). Conviene observar que, si solo se emplean traslaciones, es necesario calcular previamente las coordenadas de las 21 cavidades que conforman los puntos de las caras. En cambio, las rotaciones y las simetrías permiten agilizar la programación por bloques, al mismo tiempo que abren la puerta a facilitar la parametrización. En la Tabla 2 desglosamos cuántos bloques de cada tipo ha empleado cada equipo.

\begin{tabular}{lccccccccc} 
& E1 & E2* & E3 & E4 & E5 & E6 & E7 & E8* & E9 \\
\hline Traslaciones & 21 & 8 & 21 & 22 & 21 & 21 & 29 & 14 & 21 \\
Rotaciones & 0 & 1 & 12 & 0 & 2 & 0 & 4 & 0 & 5 \\
Simetrías (mirror) & 0 & 0 & 0 & 0 & 3 & 0 & 3 & 0 & 0 \\
Esferas & 23 & 1 & 22 & 23 & 22 & 22 & 23 & 15 & 22 \\
Cubos & 1 & 1 & 1 & 1 & 0 & 1 & 1 & 1 & 1 \\
Cilindros & 0 & 8 & 0 & 0 & 3 & 0 & 0 & 0 & 0 \\
Color & 0 & 8 & 1 & 0 & 0 & 0 & 0 & 0 & 21 \\
Intersecciones & 0 & 1 & 1 & 1 & 1 & 1 & 1 & 1 & 1 \\
Uniones & 0 & 0 & 0 & 0 & 5 & 0 & 11 & 0 & 15 \\
Diferencias & 3 & 0 & 1 & 22 & 1 & 21 & 1 & 1 & 1 \\
Lados (sides) & 0 & 0 & 0 & 22 & 0 & 21 & 21 & 0 & 0
\end{tabular}

Tabla 2. Tipo y número de bloques empleados por cada uno de los equipos participantes. Los modelos de E2 y E8 están incompletos.

Aunque la Tabla 2 deja entrever la variedad de soluciones que aportan los participantes, así como el grado de eficiencia de su código, las rotaciones y simetrías no son empleadas por todos Ios equipos de la misma manera. El equipo E3, por ejemplo, ofrece una configuración alternativa (Figura 8) interesante, puesto que las cavidades de las caras tres y seis las ubican a lo largo de una circunferencia, de manera que la separación angular entre ellas sea 360/N. Para las cavidades de las caras cuatro y cinco proceden de la misma manera, pero colocan una cavidad en el centro. $Y$ las caras uno y dos son singulares.
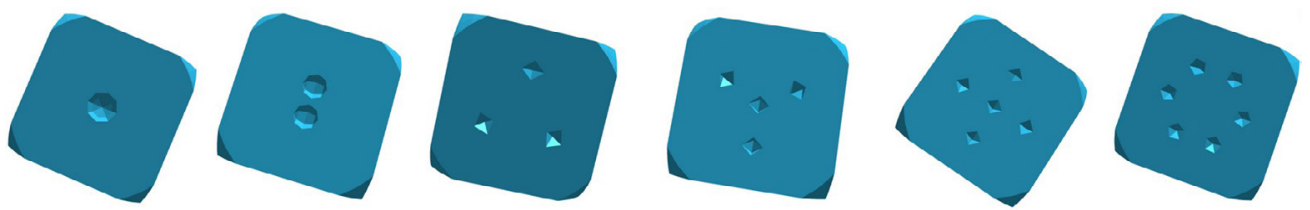

Figura 8. Con iguraciones puntuales para el valor de cada dado (E3). 
La rotación que aparece en la producción, incompleta, del E2 puede ser un intento de utilizar rotaciones para facilitar la colocación de las cavidades, pero no es posible asegurarlo.

Tres de los equipos (E5, E7, E9) emplean uniones para unir en un solo cuerpo grupos de objetos y posteriormente manipularlo, rotarlo o utilizarlo en una diferencia. Un fenómeno que hemos observado es que hay equipos que utilizan difference de forma binaria, cuando realmente permite restar varios objetos a un objeto principal. En la Tabla 2, esto se refleja en que hay equipos con un bloque difference, y otros con 21 o 22.

El equipo E2 emplea bloques color para resaltar en color negro los cilindros salientes que configuran el valor de cada cara, mientras que E9 hace lo mismo para resaltar las superficies late-rales de las cavidades. E3 también emplea el bloque color, pero lo aplica a todo lo que hace, cam-biando el color por defecto.

Algunos equipos (E4, E6, E7) manifestaron problemas a la hora de renderizar los modelos, por lo que utilizaron el bloque sides para forzar a BlocksCAD a ofrecer una representación visual más fiel a lo que tenían en mente. De lo contrario, las renderizaciones podían mostrar la base circular de un cilindro con cuatro o cinco segmentos, técnica utilizada para reducir la carga computacional en el servidor. Por otro lado, ninguno de los equipos hace uso de funcionalidades avanzadas propias de BlocksCAD, como el uso de módulos para hacer un código más eficiente. No obstante, esto es debido a que no se ha formado específicamente en la herramienta y está relacionado con las dificultades que expresaron, aspecto que comentamos en el siguiente punto.

\subsection{Dificultades manifestadas en la realización de esta tarea}

Podemos distinguir dos grandes tipos de dificultades. Por un lado, aquellas que tienen que ver con el empleo de la herramienta $y$, por otro lado, aquellas relacionadas con la complejidad de las tareas matemáticas involucradas. En la Tabla 3 recogemos todas las dificultades que expresaron los par-ticipantes.

Debido a que no hubo formación previa en la herramienta, más allá del taller introductorio que se hizo durante la sesión, varios equipos manifestaron que el tiempo fue escaso y/o que no había habido formación específica, previa, sobre BlocksCAD. Tres de los equipos (E4, E5, E7) men-cionan que el proceso de realizar las cavidades de cada cara fue tedioso. Esto, en realidad, es algo relacionado con la herramienta, ya que permite utilizar módulos (bloque module) para producir código de manera más eficaz. E7 alude a esta cuestión y, en el mismo sentido, E4 fue bastante explícito:

E7: Problema: la interfaz es cómoda para objetos simples, pero se hace pesada para configuraciones complejas. Poco flexible.

E4: El principal problema ha sido el desconocimiento del interfaz y de procedimientos adecuados que permitieran agilizar la realización de tareas repetitivas (traslaciones de los puntos)

Uno de los equipos (E1) menciona como dificultad el tiempo de renderizado de los modelos, lo cual puede indicar cierta inseguridad a la hora de trabajar con esta herramienta, ya que permitiría ir trabajando sin necesidad de renderizar cada bloque que se añade. Y otro equipo (E6) menciona 
que el sistema de arrastrar y soltar bloques no les funcionaba del todo bien, lo cual pudo deberse a cuestiones técnicas de su dispositivo.

La dificultad más común relacionada con las matemáticas necesarias para la realización de la tarea fue la derivada de las consideraciones y cálculos necesarios para colocar las cavidades en las posiciones deseadas. Esto fue lo que expresaron cuatro de los equipos (E1, E2, E3, E7). Los equipos E1 y E2 son bastante explícitos al señalar que la fuente de su dificultad es la visión espacial:

E1: A veces las esferas no salían donde queríamos.

E2: Los problemas surgen al ubicar los puntos, ya que probablemente por falta de visión espacial, resulta muy difícil colocar los puntos en el lugar adecuado.

El equipo E2, por otro lado, manifiesta que tuvieron dificultades con las operaciones lógicas. Al tratar de suavizar las esquinas del dado, inicialmente pensaron en hacer la diferencia entre cubo y esfera, cuando realmente eso se hacía con la intersección:

E2: Otro problema ha sido a la hora de "curvar" las esquinas del dado. Ya que pensábamos que era haciendo la diferencia del cubo y esfera, pero es con la intersección de estas dos figuras en 3D.

Otra dificultad que señala el equipo E2 es que no se pueden poner alturas negativas en el cilindro. Hemos decidido no clasificar esta dificultad en ninguna de las categorías anteriores, ya que incluye elementos de ambas (conocimiento de BlocksCAD y conocimiento matemático):

E2: Hemos tenido problemas para ver los puntos en las coordenadas negativas porque no se consideran alturas negativas del cilindro y hemos tenido que jugar con la altura.

Finalmente, el equipo E9 no expresa ninguna dificultad.

\begin{tabular}{|c|c|c|c|c|c|c|c|c|c|c|}
\hline \multirow{3}{*}{$\begin{array}{l}\text { Relacionadas con la } \\
\text { herramienta }\end{array}$} & & E1 & E2 & E3 & E4 & E5 & E6 & E7 & E8 & E9 \\
\hline & $\begin{array}{l}\text { Tiempo de renderizado } \\
\text { excesivo en ocasiones }\end{array}$ & $\mathrm{x}$ & & & & & & & & \\
\hline & Tediosidad & & & & $\mathrm{x}$ & $\mathrm{x}$ & & $\mathrm{x}$ & & \\
\hline & Poco tiempo disponible & & & & & $\mathrm{x}$ & & & $\mathrm{x}$ & \\
\hline & $\begin{array}{l}\text { Poca formación en la } \\
\text { herramienta }\end{array}$ & & & & & $\mathrm{x}$ & & & $\mathrm{x}$ & \\
\hline & Sistema de arrastra y suelta & & & & & & $\mathrm{x}$ & & & \\
\hline $\begin{array}{l}\text { Relacionadas con la } \\
\text { matemáticas }\end{array}$ & $\begin{array}{l}\text { sCálculo de las posiciones de } \\
\text { los puntos de cada cara }\end{array}$ & $\mathrm{x}$ & $\mathrm{x}$ & $\mathrm{x}$ & & & & $\mathrm{x}$ & & \\
\hline$\underline{\text { necesarias }}$ & Operaciones lógicas & & $\mathrm{x}$ & & & & & & & \\
\hline Sin clasificar & Altura negativa cilindro & & $\mathrm{x}$ & & & & & & & \\
\hline
\end{tabular}

Tabla 3. Dificultades que expresaron los equipos. 


\subsection{Propuestas de secuencias didácticas}

Ante la tarea "Diseña una secuencia de actividades para secundaria inspirada en esta tarea, estableciendo conexiones intra y extra-matemáticas", todos los grupos plantean una secuencia de actividades en las que, al menos, incluyen el modelado de distintos objetos con el programa BlocksCAD. Por tanto, surgen conexiones interdisciplinares con tecnología e informática, de manera explícita en el caso de E6, E7 y E8, y de manera implícita, en el resto de los equipos. Por otro lado, todos los equipos, salvo E5, plantean actividades de experimentación con dados sesgados por lo que aparecen conexiones intra-disciplinares con contenidos matemáticos como la probabilidad con significado frecuencial.

A continuación, nos centramos en abordar la descripción de las actividades enunciadas (ver Tabla 4). En primer lugar, hay que indicar que ningún equipo contextualizó las tareas en algún curso concreto de educación secundaria. Como hemos señalado anteriormente, todos los equipos, excepto uno, plantean actividades con dados sesgados, con tiradas sucesivas o en el contexto de un juego, donde hay que identificar o averiguar si un dado posee sesgo o no. Además, E3, E6, E7 y E9 plantean que, una vez identificado los sesgos del dado por parte de los estudiantes, se investigue el origen de estos en el diseño de los dados.

La actividad análoga a la realizada en la sesión, como el diseño de dados con BlocksCAD, bien idénticos al realizado o con distintas formas, están presentes en las respuestas de seis equipos (E3, E4, E6, E7, E8 y E9) donde, la mayoría de ellos, además, plantean la fabricación efectiva de los dados en una impresora 3D. Los otros objetos diseñados con BlocksCAD son de naturaleza variada, unos más cercanos a las matemáticas (modelos de cuerpos geométricos de poliedros regulares, en el caso de E2 y E5), cercanos a otras disciplinas (modelos de moléculas, cadenas de ADN, cristalográficos, o del sistema solar, como E2 y E8), o del entorno del estudiante (señales de tráfico y un balón de fútbol, en el caso de E1 y E5).

Finalmente, hemos de señalar que en algunos pocos equipos aparecen otros tipos de actividades no relacionadas directamente con los temas anteriores como averiguar experimentalmente el volumen de cuerpos mediante trasvasado (E5), o realizar una investigación histórica sobre el juego de las tabas y realizar una representación artística del dado mediante un esbozo, una fotografía retocada o un retoque digital de la captura de la imagen en pantalla (E7).

\begin{tabular}{|c|c|c|c|c|c|c|c|c|c|c|}
\hline \multirow{3}{*}{ Dados BlocksCAD } & & E1 & E2 & E3 & E4 & E5 & E6 & E7 & E8 & E9 \\
\hline & Diseño & & & $\mathrm{x}$ & $\mathrm{x}$ & & $\mathrm{x}$ & $\mathrm{x}$ & $\mathrm{x}$ & $\mathrm{x}$ \\
\hline & Construcción & & & $\mathrm{x}$ & & & $\mathrm{x}$ & $\mathrm{x}$ & $\mathrm{x}$ & $\mathrm{x}$ \\
\hline \multirow[t]{2}{*}{ Objetos BlocksCAD } & Diseño & $\mathrm{x}$ & $\mathrm{x}$ & & & $\mathrm{x}$ & & & $\mathrm{x}$ & \\
\hline & Construcción & $\mathrm{x}$ & $\mathrm{x}$ & & & $\mathrm{x}$ & & & & \\
\hline \multicolumn{2}{|c|}{ Experimentación con dados sesgados } & $\mathrm{x}$ & $\mathrm{x}$ & $\mathrm{x}$ & $\mathrm{x}$ & & $\mathrm{x}$ & $\mathrm{x}$ & $\mathrm{x}$ & $\mathrm{x}$ \\
\hline
\end{tabular}

Tabla 4. Tipos de actividades en la secuencia diseñada por los equipos. 
En cuanto a las conexiones, a pesar del enunciado de la tarea, solo cinco equipos mencionan explícitamente las conexiones intra y extra-matemáticas realizadas (E2, E5, E6, E7 y E8), mientras que los demás equipos, o bien no saben establecer conexiones, o bien asumen que es obvio apreciarlas en el diseño de las actividades planteadas.

Además de la conexión obvia con la materia de Tecnología, una de las conexiones interdisciplinares más ricas que ofrece esta actividad es la conexión con la materia de Física. En este sentido, únicamente los equipos E6 y E7 relacionan la actividad del estudio de sesgos desde un punto de vista físico, utilizando la idea de centro de gravedad.

Los equipos E3, E4 y E9 únicamente establecen conexiones "obvias", de manera implícita, mencionadas anteriormente, con la probabilidad y la tecnología.

E1 y E5 plantean conexiones con el entorno del entorno mediante el modelado de objetos tales como señales de tráfico y un balón de fútbol, respectivamente. E5 menciona de manera explícita que el modelado del balón es una conexión con la materia de Educación Física. Sin embargo, los contenidos que se movilizan serían los mismos que resultarían de modelar cualquier otro objeto, y no se pone en juego nada significativo de la materia de Educación Física en la etapa.

Algo similar ocurre con los equipos E2 y E8, que proponen el modelado de objetos 3D con cierto componente geométrico (sistema solar, moléculas, cristales, etc.) presentes en otras disciplinas como Física, Química, Biología, Geología, etc., explicitando de esta manera una conexión interdisciplinar. Mientras que el equipo E8 al plantear la actividad del sistema solar, alude a la necesidad de que el alumnado utilice proporciones y escalas; las actividades sobre moléculas y cristales de E2 únicamente son tareas de modelado, sin integrar materias y establecer conexiones.

En cuanto a los equipos E7 y E8, relacionan la actividad con la materia de Educación Plástica (dibujo técnico), estableciendo de esta manera conexiones interdisciplinares. No obstante, no desarrollan mucho la actividad, limitándose, en el caso de E7, a "Realizar una representación artística del dado: fotográfica, esbozo, pantallazo de blockscad y retocado".

Finalmente, el equipo E7 establece conexiones con la asignatura de Historia mediante la propuesta de una investigación histórica sobre un juego de azar donde se emplean dispositivos aleatorios (mencionan el juego de las tabas, a modo de "dados").

\section{Conclusiones}

La experiencia formativa que se describe constituye un ejemplo que conjuga conocimientos y competencias matemáticas (acerca de la geometría, la visualización y el pensamiento computacional), con conocimientos y competencias tecnológicas y con la competencia docente de diseño de tareas. Al mismo tiempo, se establecen conexiones intra e interdisciplinares y con el entorno. Se trata, por tanto, de una experiencia a considerar con estudiantes de másteres de profesorado, donde el tiempo que se dispone es limitado y hay que dividirlo entre conocimiento del contenido y conocimiento didáctico. 
El modelado del dado es la parte de la tarea que únicamente requiere conocimientos y competencias matemáticas y tecnológicas. Si bien los autores somos conscientes de que no hubo formación previa sobre el uso de la herramienta, en un contexto de estudiantes de máster, esto no resulta siempre factible. No obstante, aunque el taller introductorio fue muy satisfactorio, ya que la mayoría de los participantes completaron el modelado, una línea de trabajo evidente consiste en realizar experiencias similares con grupos de alumnos ya instruidos en el uso de BlocksCAD.

Los modelados a los que llegan los participantes son diversos. La principal diferencia ha sido el empleo de más o menos tipos de bloques de BlocksCAD, cuestión que se refleja en la síntesis que permite el empleo de rotaciones y simetrías, al evitar el cálculo de coordenadas innecesarias y facilitar la parametrización. También nos hemos encontrado con tres maneras de modelar el cuerpo del dado: como la intersección de un cubo con una esfera, como la diferencia de un cubo con una corona esférica o como la intersección de tres cilindros y una esfera.

En el análisis de las producciones se identificaron algunos de los mismos sesgos que se observaron en una implementación anterior de la tarea del dado con alumnado de educación secundaria (Beltrán-Pellicer, 2017). Así, algunos participantes no compensan el material retirado haciendo que las caras opuestas sumen siete, como en los dados estándar. Uno de los equipos, además, en lugar de hacer cavidades incluye salientes.

Durante la realización de la tarea, los participantes tienen ocasión de reflexionar sobre las posibles actividades que se pueden plantear al alumnado de ESO con esta herramienta. El hecho de haber modelado un dado, las conexiones intra-disciplinares (intra-matemáticas) que proponen los participantes están orientadas a la probabilidad o a la visualización espacial. La naturaleza de la tarea planteaba conexiones evidentes entre Matemáticas y Tecnología, por lo que muchos equipos han propuesto tareas de modelado de diferentes cuerpos en este sentido. En menor medida, hemos observado que algunos estudiantes son capaces de establecer conexiones interdisciplinares con materias como Física, Educación Plástica, Biología, Geología, Educación Física e Historia. Ahora bien, el grado de significatividad curricular es muy variado, y solamente dos equipos, por ejemplo, proponen un análisis detallado de los sesgos desde la física. Finalmente, solo unos pocos participantes proponen actividades que indiquen conexiones con el entorno y la vida real.

Algunas administraciones educativas españolas han visto el trabajo por "ámbitos científicos" en los primeros cursos de educación secundaria como una medida para afrontar las nuevas problemáticas educativas que se han producido fruto de la crisis sanitaria producida por la COVID y como reclamo para fomentar la educación STEM. La escasez de conexiones establecidas (solo cinco de los nueve equipos las explicitan) y la calidad de algunas de éstas por parte de los profesores en formación señala que plantear este tipo de trabajo por ámbitos está lejos de ser una tarea profesional sencilla para los docentes y muestra la necesidad de que el profesorado adquiera una formación sólida en ámbito STEM. Esta formación trasciende el hecho de poseer amplios conocimientos matemáticos, científicos o tecnológicos, sino que los profesores también deben ser capaces de integrar las disciplinas y de identificar conexiones entre las mismas para que los estudiantes puedan alcanzar la adquisición de competencias y las mejoras en rendimiento académico y creencias sobre las materias, en línea con lo apuntado por McDonald (2016) y Diego-Mantecón et al. (2021). 


\section{Agradecimientos}

Este trabajo se ha desarrollado dentro del proyecto PID2019-105601GB-I00 / AEI / 10.13039/501100011033 y del grupo S60_20R - Investigación en Educación Matemática (Gobierno de Aragón y Fondo Social Europeo).

\section{Referencias}

Alsina, Á. (2020). Conexiones matemáticas a través de actividades STEAM en Educación Infantil. Unión, 16(58), 168-190. https://union.fespm.es/index.php/UNION/article/download/69/30/

Baeza-Alba, M. A., Claros-Mellado, F.J. y Sánchez-Campaña, M.T. (2016). Una propuesta didáctica en $3^{\circ}$ ESO para trabajar el pensamiento matemático avanzado haciendo uso de Scratch. Épsilon, 33(2), 31-46. https://bit.ly/3rXehAb

Beltrán-Pellicer, P. (2017). Modelado e impresión 3D como recurso didáctico en el aprendizaje de la probabilidad. Épsilon, 34(95), 99-106. http://funes.uniandes.edu.co/17047/

Beltrán-Pellicer, P. y Rodríguez-Jaso, C. (2017). Modelado e impresión en 3D en la enseñanza de las matemáticas: un estudio exploratorio. ReiDoCrea, 6, 16-28. http://hdl.handle.net/10481/44193

Beltrán-Pellicer, P. y Rodríguez-Jaso, C. (2018). Construcciones en BlocksCAD para analizar el conocimiento en geometría. 1.er Workshop sobre Entornos Tecnológicos en Educación Matemática, Valencia, España.

Beltrán-Pellicer, P., Rodríguez-Jaso, C. y Muñoz-Escolano, J.M. (2020). Introduciendo BlocksCAD como recurso didáctico en matemáticas. SUMA, 93, 39-48. https://bit.ly/3Ah2aka

Bush, S. B., Cox, R. y Cook, K. L. (2016). A critical focus on the M in STEAM. Teaching Children Mathematics, 23(2), 110-114. https://bit.ly/37lZip'

Carmona-Mesa, J. A., Cardona Zapata, M. E. y Castrillón-Yepes, A. (2020). Estudio de fenómenos físicos en la formación inicial de profesores de Matemáticas. Una experiencia con enfoque STEM. Uni-Pluriversidad, 2O(1), 18-38. https://doi.org/10.17533/udea.unipluri.20.1.02

Chytas, C., Diethelm, I. y Tsilingiris, A. (2018). Learning programming through design: An analysis of parametric design projects in digital fabrication labs and an online makerspace. En 2018 IEEE Global + Engineering Education Conference (EDUCON) (pp. 1978-1987). IEEE.

Dickson, B., Weber, J., Kotsopoulos, D., Boyd, T., Jiwani, S. y Roach, B. (2020). The role of productive failure in $3 \mathrm{D}$ printing in a middle school setting. International Journal of Technology and Design Education, 31, 489-502. https://doi.org/10.1007/s10798-020-09568-Z

Diego-Mantecón, J. M., Arcera, O., Fernández-Blanco, T. y Lavicza, Z. (2019). An engineering technology problemsolving approach for modifying student mathematics-related beliefs: Building a robot to solve a Rubik's cube. International Journal for Technology in Mathematics Education, 26(2), 55-64.

http://blogs.exeter.ac.uk/ijtme/vol-26-no-2-2019/

Diego-Mantecón, J., Fernández-Blanco, T., Ortiz-Laso, Z. y Lavicza, Z. (2021). STEAM projects with KIKS format for developing key competences. [Proyectos STEAM con formato KIKS para el desarrollo de competencias clave]. Comunicar, 66, 33-43. https://doi.org/10.3916/C66-2021-03

Ferrer, T. (2011). Usando Scratch en secundaria. Competencia matemática y aprender a aprender. Aula de Innovación Educativa, 206, 20-23. https://www.grao.com/es/producto/usando-scratch-en-secundaria

Freudenthal, H. (1991). Revisiting mathematics education. Kluwer Academic Publishers.

Gleasman, C. y Kim, C. (2020). Pre-service teacher's use of block-based programming and computational thinking to teach elementary mathematics. Digital Experiences in Mathematics Education, 6, 52-90. https://doi.org/10.1007/s40751-019-00056-1 
Godino, J.D., Batanero, C., Font, V. y Giacomone, B. (2016). Articulando conocimientos y competencias del profesor de matemáticas: el modelo CCDM. En J.A. Macías, A. Jiménez, J.L. González, M.T. Sánchez, P. Hernández, C. Fernández, F.-J. Ruiz, T. Fernández y A. Berciano (Eds.), Investigación en Educación Matemática $X X$ (pp. 285-294). SEIEM.

González-Gómez, J., Valero-Gómez, A., Prieto-Moreno, A. y Abderrahim, M. (2012). A new open source 3D-printable mobile robotic platform for education. Advances in autonomous mini robots (pp. 49-62). Springer.

Gutiérrez, Á. y Jaime, A. (2015). Análisis del aprendizaje de geometría espacial en un entorno de geometría dinámica 3-dimensional. PNA, 9(2), 53-83. https://doi.org/10.30827/pna.vgi2.6106

Jones, R., Haufe, P., Sells, E., Iravani, P., Olliver, V., Palmer, C. y Bowyer, A. (2011). RepRap-the replicating rapid prototyper. Robotica, 29(1), 177-191.

Hernández, S., Fernández, C. y Baptista, L. (2010). Metodología de la investigación. Mc Graw Hill. Hohenwarter, M. y Fuchs, K. (2004). Combination of dynamic geometry, algebra and calculus in the software system

GeoGebra. En Computer algebra systems and dynamic geometry systems in mathematics teaching conference (pp. 128-133). Bornus Nyomda.

Hoyles, C. y Lagrange, J. B. (Ed.) (2010). Mathematics education and technology: Rethinking the terrain. Springer.

Jorge-Pozo, D., Jiménez-Gestal, C. y Murillo, J. (2017). Influencia de un entorno virtual de enseñanza aprendizaje en la afectividad hacia las matemáticas de estudiantes de secundaria: estudio de casos. En J. M. MuñozEscolano, A. Arnal-Bailera, P. Beltrán-Pellicer, M. L. Callejo y J. Carrillo (Eds.), Investigación en Educación Matemática XXI (pp. 325-334). SEIEM.

Martín-Páez, T., Aguilera, D., Perales-Palacios, F. J. y Vílchez-González, J. M. (2019). What are we talking about when we talk about STEM education? A review of literature. Science Education, 103, 799-822.

https://doi.org/10.1002/sce.21522

Ministerio de Educación Cultura y Deporte (MECD) (2015). Real Decreto 1105/2014, de 26 de diciembre, por el que se establece el currículo básico de la Educación Secundaria Obligatoria y del Bachillerato. BOE, 3 (pp. 169-546).

McDonald, C. V. (2016). STEM Education: A review of the contribution of the disciplines of science, technology, engineering and mathematics. Science Education International, 27(4), 530-569.

http://www.icaseonline.net/sei/december2016/p4.pdf

Mishra, P. y Koehler, M. J. (2006). Technological pedagogical content knowledge: A framework for teacher knowledge. Teachers College Record, 108(6), 1017-1054. http://one2oneheights.pbworks.com/f/MISHRA PUNYA.pdf

NCTM (2000). Principles and standards for school mathematics.

$\mathrm{Ng}$, O. L. (2017). Exploring the use of 3D computer-aided design and 3D printing or STEAM learning in mathematics. Digital Experiences in Mathematics Education, 3(3), 257-263.

https://doi.org/10.1007/s40751-017-0036-X

Ng, O. L. y Chan, T. (2019). Learning as Making: Using 3D computer-aided design to enhance the learning of shape and space in STEM-integrated ways. British Journal of Educational Technology, 50(1), 294-308. https://doi.org/10.1111/bjet.12643

Ortega, T. (2005). Conexiones matemáticas. Motivación del alumnado y competencia matemática. Graó.

Papert, S. (1980). Mindstorms: Children, computers, and powerful ideas. Basic Books.

Rocard, M., Csermely, P., Jorde, D., Lenzen, D., Walwerg Henriksson, H. Y. y Hemmo, V. (2007). Science education now: a renewed pedagogy for the future of Europe. Office for Official Publications of the European Communities.

Schelly, C., Anzalone, G., Wijnen, B. y Pearce, J. M. (2015). Open-source 3-D printing technologies for education: Bringing additive manufacturing to the classroom. Journal of Visual Languages \& Computing, 28, 226237. https://doi.org/10.1016/j.jvlc.2015.01.004 
Shaughnessy, J. M. (2013). Mathematics in a STEM context. Mathematics Teaching in the Middle school, 18(6), 324-324. https://doi.org/10.5951/MATHTEACMIDDSCHO.18.6.0324

Stohlmann, M. (2020). STEM integration for high school Mathematics teachers. Journal of Research in STEM Education, 6(1), 52-63. https://doi.org/10.51355/jstem.2020.71

Wang, T. H., Lim, K. Y., Lavonen, J. y Clark-Wilson, A. (2019). Maker-centred science and mathematics education: lenses, scales and contexts. International Journal of Science and Mathematics Education, 17(1), 1-11. https://doi.org/10.1007/s10763-019-09999-8

Weintrop, D. y Wilensky, U. (2015). To Block or not to block, That is the question: Students' perceptions of blocks-based programming. En Proceedings of the 14th international conference on interaction design and children (pp. 199-208). Association for Computing Machinery, Inc. https://doi.org/10.1145/2771839.2771860

Wing, J. M. (2006). Computational thinking. Communications of the ACM, 49(3), 33-35.

https://doi.org/10.1145/1118178.1118215 